\title{
ON NONLINEAR BOUNDARY VALUE PROBLEMS WITH DEVIATING ARGUMENTS AND DISCONTINUOUS RIGHT HAND SIDE ${ }^{1}$
}

\author{
B.C. DHAGE \\ Mahatma Gandhi Mahavidyalaya \\ Department of Mathematics \\ Ahmedpur-413515, INDIA \\ S. HEIKKILÄ \\ University of Oulu \\ Department of Mathematics \\ SF-90570 Oulu 57, FINLAND
}

\begin{abstract}
In this paper we shall study the existence of the extremal solutions of a nonlinear boundary value problem of a second order differential equation with general Dirichlet/Neumann form boundary conditions. The right hand side of the differential equation is assumed to contain a deviating argument, and it is allowed to possess discontinuities in all the variables. The proof is based on a generalized iteration method.
\end{abstract}

Key words: Nonlinear boundary value problems, deviating arguments, discontinuous right-hand side.

AMS (MOS) subject classifications: $\quad 34 \mathrm{~K} 10,47 \mathrm{H} 07,47 \mathrm{H} 10$.

\section{INTRODUCTION}

Let $\mathbb{R}$ denote the set of all real numbers and $\mathbb{R}_{+}$the set of all nonnegative reals. Given a real interval $I=\left[t_{0}, t_{1}\right], t_{0}<t_{1}$, consider the differential equation with a deviating argument

$$
L x(t)=f(t, x(t), x(\varphi(t)) \text { a.e. on } I
$$

where $\varphi$ is a continuous real function on $I$, and $f: I \times \mathbb{R}^{2} \rightarrow \mathbb{R}$. Choosing an interval $J=\left[s_{0}, s_{1}\right]$ which contains both $I$ and $\varphi[I]$, and denoting $I_{0}=\left[s_{0}, t_{0}\right]$ and $I_{1}=\left[t_{1}, s_{1}\right]$, we shall adjoin to the Equation (1.1) the following boundary conditions:

\footnotetext{
${ }^{1}$ Received: December, 1992. Revised: March, 1993.
} 
where

$$
B_{j} x(t)=a_{j} x(t)-(-1)^{j} b_{j} x^{\prime}(t)=\alpha_{j}(t), \quad t \in I_{j}, \quad j=0,1
$$

(i) $\quad a_{j}, b_{j} \in \mathbb{R}_{+}, a_{j}+b_{j}>0$, and $\alpha_{j} \in L^{1}\left(I_{j}\right), j=0,1$.

The operator $L: A C^{1}(I) \rightarrow L^{1}(I)$ has the form

(ii) $L x=-x^{\prime \prime}+p x^{\prime}+q x$, where $p, q \in L^{\infty}(I)$ and $q$ is positive-valued.

A function $x \in C(J)$ is said to be a lower solution of the BVP (1.1)-(1.2) if its first derivative exists and is absolutely continuous on $I$, and if

$$
L x(t) \leq f\left(t, x(t), x(\varphi(t)) \text { a.e. on } I, \text { and } B_{j} x(t) \leq \alpha_{j}(t), t \in I_{j}, j=0,1 .\right.
$$

$x$ is called an upper solution of (1.1)-(1.2) if the reversed inequalities hold in (1.3). If equalities hold in (1.3), we say that $x$ is a solution of $(1.1)-(1.2)$.

In the case when $f$ is zero function, the problem (1.1)-(1.2) has a unique solution which we denote by $z$. In the special case when $f$ is a function of $t$ alone, it can be shown that the problem $(1.1)-(1.2)$ has at most one solution in the class $A C^{1}(I)$. The existence of the solution is guaranteed by the above regularity and boundary conditions, and can be extended via boundary conditions to a unique solution of $(1.1)-(1.2)$ in the sense defined above.

The existence and uniqueness of the solution to problem (1.1) - (1.2) is discussed in $[1,2]$ by using classical comparison and iteration methods, and assuming that $f$ is a continuous function in all its three arguments. Recently, the existence of extremal solutions to the BVP $(1.1)-(1.2)$ is studied in [3] in the special case without deviating arguments, but allowing discontinuous nonlinearity for $f$, by using a generalized iteration method. In the present paper we shall study the existence of the extremal solutions of the general problem $(1.1)-(1.2)$ between given lower and upper solutions via a generalized iteration method developed in [3, 4], and a comparison method. The function $f$ is required to be continuous only at the points $\left(t_{j}, x, y\right), j=0,1$.

\section{PRELIMINARIES}

Let $C_{+}(J)$ denote the space of all nonnegative-valued functions of $C(J)$. We assume that the space $C(J)$ is endowed with the norm $\|\cdot\|_{0}$ and with the partial ordering $\leq$ defined by

$$
\|x\|_{0}=\max _{t \in J}|x(t)|, \text { and } x \leq y \text { if and only if } x(t) \leq y(t) \text { for all } t \in J .
$$

If, $a, b \in C(J)$, denote $[a, b]=\{x \in C(J) \mid a \leq x \leq b\}$. 
We shall impose the following assumptions on the function $f: I \times \mathbb{R}^{2} \rightarrow \mathbb{R}$ :

(f0) The BVP (1.1) - (1.2) has a lower solution $a$ and an upper solution $b$ such that $a \leq b$.

(f1) $f(\cdot, x(\cdot), y(\cdot))$ is measurable for all $x, y \in[a, b]$.

(f2) There exists $h \in L_{+}^{\infty}(I)$ such that $(x, y) \mapsto f(t, x, y)+h(t) x$ is nondecreasing in $[a,(t), b(t)] \times[a(\varphi(t)), b(\varphi(t))]$ for almost all $t \in I$.

(f3) $f$ is continuous at $\left(t_{j}, x, y\right)$ for all $x \in\left[a\left(t_{j}\right), b\left(t_{j}\right)\right]$ and $y \in\left[a\left(\varphi\left(t_{j}\right)\right), b\left(\varphi\left(t_{j}\right)\right)\right]$, $j=0,1$.

(f4) The functions $f(\cdot, a(\cdot), a(\varphi(\cdot))$ and $f(\cdot, b(\cdot), b(\varphi(\cdot))$ are Lebesgue integrable.

Consider now the BVP

$$
\mathcal{L} x(t)=g\left(t, y(t), y(\varphi(t)) \text { a.e. on } I, B_{j} x(t)=\alpha_{j}(t), t \in I_{j}, j=0,1\right.
$$

where

$$
\mathcal{L} x=L x+h(t) x \text { and } g(t, x, y)=f(t, x, y)+h(t) x .
$$

If $y \in[a, b]$, it follows from (f1), (f2) and (2.3) that $g(\cdot, y(\cdot), y(\varphi(\cdot))$ is measurable on $I$, that both $g(\cdot, a(\cdot), a(\varphi(\cdot))$ and $g(\cdot, b(\cdot), b(\varphi(\cdot))$ are Lebesgue integrable on $I$, and that

$$
\mid g(t, y(t), y(\varphi(t)) \mid \leq M(t) \text { for a.a. } t \in I
$$

where

$$
M(t)=2 \mid g(t, a(t), a(\varphi(t))|+| g(t, b(t), b(\varphi(t)) \mid, t \in I .
$$

Hence, the equation

$$
F y(t)=\int_{t_{0}}^{t_{1}} k(t, s) g(s, y(s), y(\varphi(s))) d s, \quad t \in I,
$$

where $k(t, s)$ is the Green's function associated with the operator $\mathcal{L}$ and the homogeneous boundary conditions

$$
a_{j} F y\left(t_{j}\right)-(-1)^{j} b_{j} F y\left(t_{j}\right)=0, j=0,1,
$$

defines a function $F y \in C(I)$ for each $y \in[a, b]$. Since $q+h \in C_{+}(\mathbb{R})$, it follows from the maximum principle that $k(t, s)$ is nonnegative on $I \times I$.

Employing the Green's function and superposition principle for ordinary linear differential equations, one can show that the BVP (2.2) has for each $y \in[a, b]$ a unique solution which can be expressed in the form 


$$
x(t)=G y(t)=z(t)+ \begin{cases}F y(t) & t \in I, \\ \exp \left(\frac{a_{j}\left(t-t_{0}\right)}{b_{j}}\right) F y\left(t_{j}\right), & t \in I_{j}, b_{j} \neq 0, j=0,1 \\ 0, & t \in I_{j}, b_{j}=0, j=0,1 .\end{cases}
$$

Lemma 2.1: The operator $G$, defined on $[a, b]$ by $(2.7)$, is nondecreasing and satisfies $a \leq G a$ and $G b \leq b$.

Proof: Let $x, y \in[a, b], x \leq y$ be given. From the definition of $g$ given by (2.3) and from (f3) it follows that $(u, v) \mapsto g(t, u, v)$ is nondecreasing in $[a(t), b(t)] \times[a(\varphi(t)), b(\varphi(t)]$ for a.a. $t \in I$. Noticing also that $k$ is nonnegative-valued, it follows from (2.5) that

$$
\begin{gathered}
F x(t)=\int_{t_{0}}^{t_{1}} k(t, s) g(s, x(s), x(\varphi(s))) d s \\
\leq \int_{t_{0}}^{t_{1}} k(t, s) g(s, y(s), y(\varphi(s))) d s=F y(t), t \in I .
\end{gathered}
$$

This and (2.7) imply that $G x \leq G y$.

Since $a$, is by (f1) a lower solution of the BVP (1.1)-(1.2), it is also a lower solution of the BVP (2.2) with $y=a$. By using this, the maximum principle (cf. [5]) and the definition of $G$, it can be shown that $a \leq G a$. Similarly, since $b$, as an upper solution of (1.1)-(1.2), is also an upper solution of (2.2) with $y=b$, it follows that $G b \leq b$.

The proof of our main result will be based on the following lemma, which can be proved by a generalized iteration method (cf. $[3,4])$.

Lemma 2.2: $\quad$ Let $[a, b]$ be a nonempty order interval in an ordered Banach space $X$, and $G:[a, b] \mapsto[a, b]$ a nondecreasing mapping. If each monotone sequence in $G[a, b]$ converges, then $G$ has the least fixed point $x_{*}$ and the greatest fixed point $x^{*}$. Moreover,

$$
x_{*}=\min \{y \in[a, b] \mid G y \leq y\}, \quad x^{*}=\max \{v y \in[a, b] \mid y \leq G y\}
$$

\section{EXISTENCE OF THE EXTREMAL SOLUTIONS}

We are now ready to prove the following result concerning the existence of the extremal solutions of the BVP $(1.1)-(1.2)$ in $[a, b]$. 
Theorem 3.1: If the hypotheses (i), (ii) and (f0)-(f4) hold, then the BVP (1.1) - (1.2) has the least and the greatest solution in the order interval $[a, b]$.

Proof: Let $a, b \in C(J)$ be as in condition (f0). From Lemma 2.1 it follows that (2.7) defines a nondecreasing mapping $G:[a, b] \rightarrow[a, b]$. In view of (2.3), (2.5) and (2.7) we have for each $y \in[a, b]$

$$
|G y(t)-G y(\bar{t})| \leq|z(t)-z(\bar{t})|+|w(t) w(\bar{t})|, y \in[a, b], t, \bar{t} \in J
$$

where

$$
w(t)= \begin{cases}\int_{t_{0}}^{t_{1}} k(t, s) M(s) d s, & t \in I, \\ \exp p\left(\frac{a_{j}\left(t-t_{0}\right)}{b_{j}}\right)\left(\left|F a\left(t_{j}\right)\right|+\left|F b\left(t_{j}\right)\right|\right), & t \in I_{j}, b_{j} \neq 0, j=0,1 \\ 0, & t \in I_{j}, b_{j}=0, j=0,1 .\end{cases}
$$

Let $\left(x_{n}\right)_{n=0}^{\infty}=\left(G y_{n}\right)_{n=0}^{\infty}$ be a monotone sequence in $G[a, b]$. Since $G[a, b] \subseteq[a, b]$, then $\left(G y_{n}(t)\right)_{n=0}^{\infty}$ is for each $t \in J$ a monotone sequence in $[a(t), b(t)]$. Thus

$$
x(t)=\lim _{n \rightarrow \infty} G y_{n}(t) \text { exist in }[a(t), b(t)] \text { for each } t \in J
$$

From (3.1) it follows that the sequence $\left(G y_{n}\right)_{n=0}^{\infty}$ is equicontinuous, whence the convergence in (3.3) is uniform. In particular, the limit functions $x$ of $\left(G y_{n}\right)_{n=0}^{\infty}$ belongs to $C(J)$.

The above proof shows that the hypotheses of Lemma 2.2 hold when $X=C(J)$, endowed with the norm and the partial ordering defined in (2.1), whence $G$ has the least fixed point $x_{*}$ and the greatest fixed point $x^{*}$. In view of the definition of $G$, both these fixed points are solutions of the BVP (2.2) with $y=x$. This and (2.3) imply that $x_{*}$ and $x^{*}$ are solutions of the BVP $(1.1)-(1.2)$ in $[a, b]$.

If $x \in[a, b]$ is a solution of $(1.1)-(1.2)$, then it satisfies the BVP (2.2) with $y=x$, whence $x$ is a fixed point of $G$. Since $x_{*}$ and $x^{*}$ are the least and the greatest fixed points of $G$, then $x_{*} \leq x \leq x^{*}$. Thus, $x_{*}$ and $x^{*}$ are the least and the greatest solutions of the BVP $(1.1)-(1.2)$ in $[a, b]$.

The conditions (f0) and (f4) can be replaced by the following growth condition:

(f5) $|f(t, x, y)| \leq H(t,|x|,|y|)$ for all $t \in I \backslash Z$ and for all $x, y \in \mathbb{R}$, where $Z$ is a null set in $I, H: I \times \mathbb{R}_{+}^{2} \rightarrow \mathbb{R}_{+}, H(t, u, v)$ is nondecreasing in $(u, v)$ for all $t \in I \backslash Z$, and the BVP

$$
L x(t)=H\left(t, x(t), x(\varphi(t)) \text { a.e. on } I, B_{j} x(t)=\left|\alpha_{j}(t)\right|, t \in I_{j}, j=0,1\right.
$$


has an upper solution in $C_{+}(J)$.

Proposition 3.1: If $f: I \times \mathbb{R}^{2} \rightarrow \mathbb{R}$ satisfies condition (f5), then condition (f0) holds for $a=-w, b=w$, where $w \in C_{+}(J)$ is an upper solution of (3.4). If conditions (f1) - (f3) hold with these $a, b$, then the BVP (1.1)-(1.2) has the extremal solutions in the order interval $[-w, w]$.

Proof: Let $\left.w \in C_{+}(J)\right)$ be an upper solution of the BVP (3.4). From (f5) it follows that

$$
\sup \{|f(t, x, y)||| x|\leq w(t),| y \mid \leq w(\varphi(t))\} \leq H(t, w(t), w(\varphi(t))) \leq L w(t) \text { for a.a. } t \in I .
$$

This implies that

$$
L(-w)(t) \leq f(t,-w(t),-w(\varphi(t))) \text { and } f(t, w(t), w(\varphi(t))) \leq L w(t) \text { a.e. on } I .
$$

Because

$$
a_{j} w\left(t_{j}\right)-(-1)^{j} b_{j} w^{\prime} l\left(t_{j}\right) \geq\left|\alpha_{j}(t)\right|, t \in I_{j}, j=0,1
$$

it follows that

$a_{j} w\left(t_{j}\right)-(-1)^{j_{b}} w^{\prime}\left(t_{j}\right) \geq \alpha_{j}(t)$, and $a_{j}(-w)\left(t_{j}\right)-(-1)^{j} b_{j}(-w)^{\prime}\left(t_{j}\right) \leq \alpha_{j}(t), t \in I_{j}, j=0,1$. Thus (f0) holds with $a=-w$ and $b=w$. If conditions (f1) -(f3) hold with these $a, b$, it follows from (3.5) and (f1) that also condition (f4) holds. Then the BVP (1.1) - (1.2) has by Theorem 3.1, the extremal solutions in the order interval $[-w, w]$.

The existence of the least and the greatest of all the solutions of the BVP $(1.1)-(1.2)$ is ensured if the condition ( $\mathrm{f} 0$ ) is replaced by

(f6) $\quad|f(t, x, y)| \leq p_{1}(t)|x|+p_{2}(t)|y|$ for all $t \in I \backslash Z$ and for all $x, y \in \mathbb{R}$, where $Z$ is a null set in $I, p_{i} \in L_{+}^{1}(I), i=1,2$, and $r(Q)<1$, where $r(Q)$ is the spectral radius of the operator $Q: C(J) \rightarrow C(J)$, defined by

$$
Q w(t)= \begin{cases}\int_{t_{0}}^{t_{1}} k(t, s)\left(p_{1}(s) w(s)+p_{2}(s) w(\varphi(s))\right) d s & t \in I, \\ \exp \left(\frac{a_{j}\left(t-t_{0}\right)}{b_{j}}\right) Q w\left(t_{j}\right), & t \in I_{j}, b_{j} \neq 0, j=0,1 \\ 0, & t \in I_{j}, b_{j}=0, j=0,1 .\end{cases}
$$

It is easy to see that (3.6) defines a bounded, linear and nondecreasing operator 
$Q: C(J) \rightarrow C(J)$. Thus the norm of $Q$ has the following representations:

$$
\|Q\|=\sup _{\|v\|_{0}=1}\|Q v\|_{0}=\|Q e\|_{0}=\max _{t \in I} Q e(t),
$$

where $e$ denotes the constant function $e(t) \equiv 1$. Each iterate $Q^{n}$ of $Q$ is also bounded, linear and nondecreasing, whence the spectral radius of $Q$ can be given by

$$
r(Q)=\lim _{n \rightarrow \infty}\left(\max _{t \in I} Q^{n} e(t)\right)=\inf _{n \in N}\left(\max _{t \in I} Q^{n} e(t)\right) .
$$

In particular, $r(Q)<1$ if and only if there exists $n \in \mathbb{N}$ such that $Q^{n} e(t)<1$ for each $t \in J$.

Proposition 3.2: If $f: I \times \mathbb{R}^{2} \rightarrow \mathbb{R}$ satisfies condition (f6), then condition (f0) holds for $a=-w, b=w$, where $w \in C_{+}(J)$ is the solution of the $B V P$

$$
\left.L w(t)=p_{1}(t) w(t)+p_{2}(t)\right) w(\varphi(t)) \text { a.e. on } I, \quad B_{j} w(t)=\left|\alpha_{j}(t)\right|, t \in I_{j}, j=0,1 \text {. }
$$

If conditions (f1)-(f3) hold with these $a, b$, then the BVP (1.1)-(1.2) has the least and the greatest solutions, and they belong to the order interval $[-w, w]$.

Proof: Denoting by $y$ the solution of (3.4) with $H \equiv 0$, the condition $r(Q)<1$ ensures that the Neumann series

$$
w=\sum_{n=0}^{\infty} Q^{n} y
$$

converges in $C_{+}(J)$ uniformly, and that the sum function $w$ is the solution of the operator equation

$$
w=y+Q w
$$

From the choice of $y$ and from the definition of $Q$ it follows that $w$ is the solution of the BVP (3.7). Thus condition (f5) holds with $H(t, u, v)=p_{1}(t) u+p_{2}(t) v$, which implies by Proposition 3.1 that (f0) holds with $a=-w$ and $b=w$. Hence, if (f1) $-(\mathrm{f} 3)$ hold, then the BVP $(1.1)-(1.2)$ has by Proposition 3.1 the extremal solutions in $[-w, w]$.

If $x$ is any solution of the BVP (1.1)-(1.2), it follows from (f6) that $x$ is a lower solution (3.10). This implies by the maximum principle (cf. [5]) that $x \leq w$. Similarly, it can be shown that $x \geq-w$. Thus the extremal solutions of $(1.1)-(1.2)$ in $[-w, w]$ are the least and the greatest of all the solutions of $(1.1)-(1.2)$.

Remark 3.1: Condition (f1) holds, for instance if $f$ coincides a.e. to a Borel measurable function in $D=\{(t, x, y) \mid a(t) \leq x \leq b(t), a(\varphi(t)) \leq y \leq b(\varphi(t))\}$.

The above results can be extended naturally with the same techniques for problems 
involving several deviating arguments, i.e. where Equation (1.1) is replaced by

$$
L x(t)=f\left(t, x(t), x\left(\varphi_{1}(t)\right), \ldots, x\left(\varphi_{m}(t)\right) \text { a.e. on } I\right.
$$

\section{AN EXAMPLE}

Let $C_{j}, \quad j=1,2,3$, be nonempty well-ordered sets in $\left[\frac{1}{2}, 1\right)$, for instance $C_{j}=\left\{\frac{1}{n+j+1} \mid j \in \mathbf{N}\right\}$. Define $g_{j}: \mathbb{R} \rightarrow \mathbb{R}, j=1,2,3$, by

$$
g_{j}(x)= \begin{cases}x, & x \in\left[0, \min C_{j}\right) \cup[1, \infty), \\ \min \left\{y \in C_{j} \cup\{1\} \mid x<y\right\}, & x \in\left[\min C_{j}, 1\right), \\ -g(-x), & x<0 .\end{cases}
$$

It is easy to see that each $g_{j}$ is nondecreasing and discontinuous at each point of $C_{j}$. Moreover, $\left|g_{j}(x)\right| \leq 2|x|$ for each $x \in \mathbb{R}$.

Choose $I=[-1,1]$, and let $p_{j} \in L_{+}^{\infty}(I), \quad j=1,2,3$, satisfy $\max \left\{\right.$ ess sup $_{j} \mid$ $j=1,2,3\} \leq 0.74$ and $g_{j}(t) \rightarrow 0$ as $t \rightarrow-1+$ or $t \rightarrow 1-$.

Consider the boundary value problem

$$
-x^{\prime \prime}(t)=f(t, x(t), x(t-1), x(t+1)), \text { a.e. on } I, x(t)=1, t \in[-2,-1] \cup[1,2]
$$

where

$$
f(t, x, y, z)=p_{1}(t) g_{1}(x)+p_{2}(t) g_{2}(y)+p_{3}(t) g_{3}(z), t \in I, x, y, z \in \mathbb{R} .
$$

It is easy to show that the function $f$ satisfies conditions (f1) - (f3). Moreover,

$$
|f(t, x, y, z)| \leq 1.48(|x|+|y|+|z|) \text {, a.e. on } I \text {. }
$$

Choosing $J=[-2,2]$, define an operator $Q: C(J) \rightarrow C(J)$ by

$Q w(t)= \begin{cases}1.48 \int_{-1}^{1} k(t, s)(w(s)+w(s-1)+w(s+1)) d s & t \in I, \\ 0, & t \in[-2,-1] \cup[1,2],\end{cases}$

where

$$
k(t, s)= \begin{cases}\frac{(1-t)(1+s)}{2}, & -1 \leq s \leq t \leq 1 \\ \frac{(1+t)(1-s)}{2}, & -1 \leq t \leq s \leq 1\end{cases}
$$

It can be shown (cf. [2]) that $r(Q)<1$. Thus $f$ satisfies also the hypothesis (f6), whence the BVP (4.2) has by Proposition 3.2 and Remark 3.1 the least and the greatest solution, and that all the solutions of (4.2) belong to the order interval $[-w, w]$, where $w$ is the 
solution of the BVP

$$
\begin{gathered}
-w^{\prime \prime}(t)=1.48(w(t)+w(t-1)+w(t+1)), t \in I \\
w(t)=1, t \in[-2,-1] \cup[1,2]
\end{gathered}
$$

\section{REFERENCES}

[1] Heikkilä, S., Mooney, J.W., and Seikkala, S., Existence, uniqueness and comparison results for nonlinear boundary value problems involving a deviating argument, $J$. Differential Equations 41, 3 (1981), 320-333.

[2] Heikkilä, S., On well-posedness of a boundary value problem involving deviating arguments, Funkcialaj Ekvacioj 28, 2 (1985), 221-232.

[3] Heikkilä, S., On fixed points through a generalized iteration method with applications to differential and integral equations involving discontinuities, Nonlinear Analysis 14, 5 (199), 413-426.

[4] Heikkilä, S., Lakshmikantham, V., and Sun, Y., Fixed point results in ordered normed spaces with applications to abstract and differential equations, J. Math. Anal. Appl. 163, 2 (1992), 422-437.

[5] Protter, M.H. and Weinberger, H.F., Maximum Principles in Differential Equations, Prentice-Hall, Englewood Cliffs, N.J. 1967. 


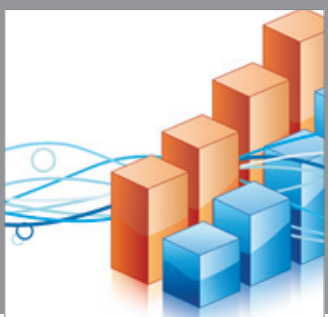

Advances in

Operations Research

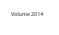

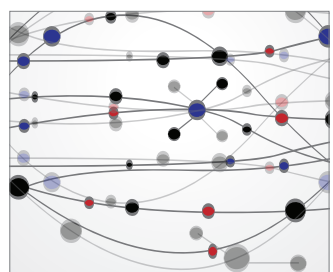

\section{The Scientific} World Journal
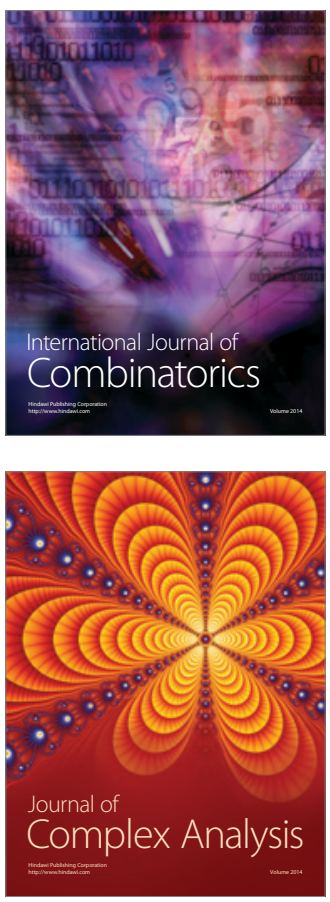

International Journal of

Mathematics and

Mathematical

Sciences
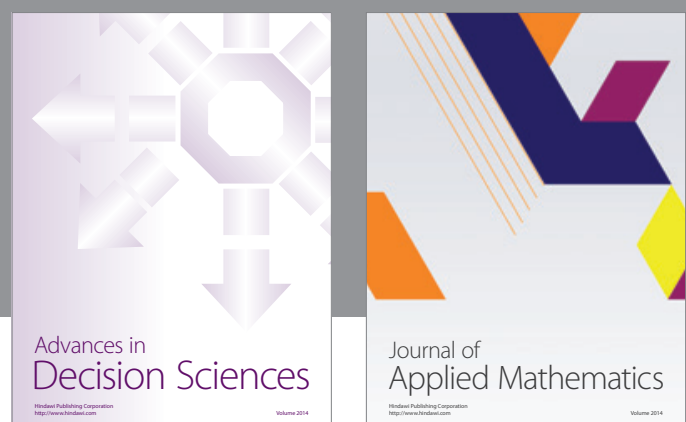

Journal of

Applied Mathematics
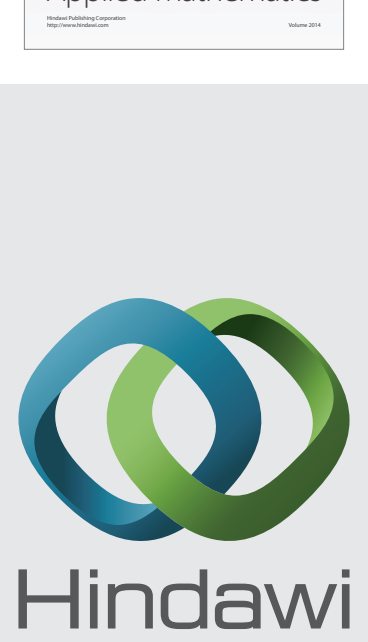

Submit your manuscripts at http://www.hindawi.com
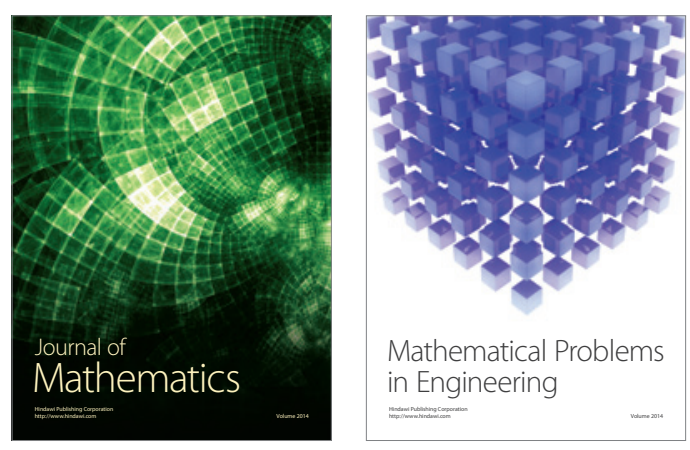

Mathematical Problems in Engineering
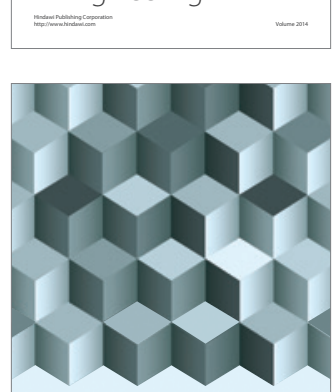

Journal of

Function Spaces
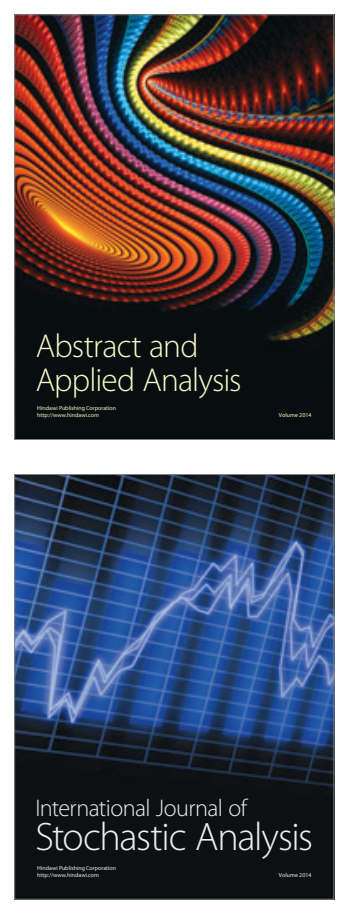

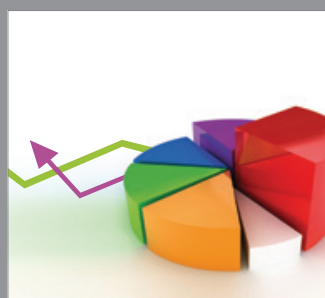

ournal of

Probability and Statistics

Promensencen
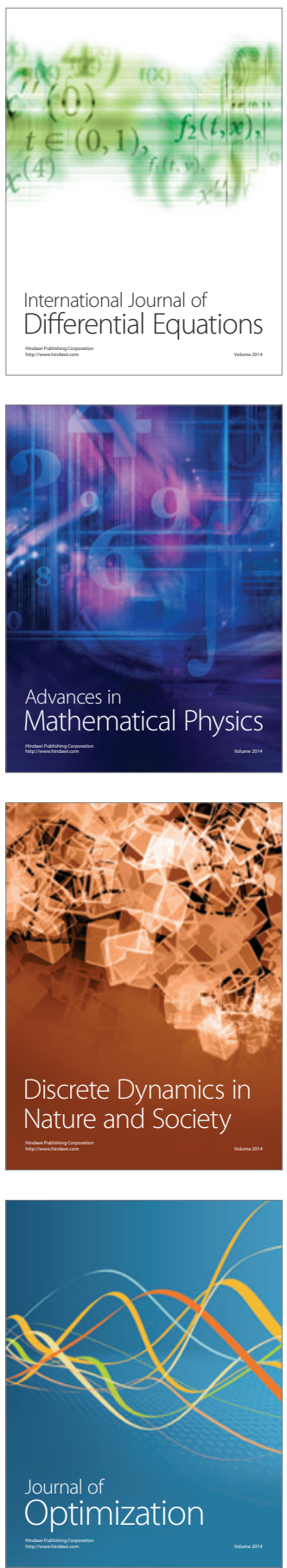